\title{
Relative stability of extended interstitial defects in silicon: First-principles calculations
}

\author{
Hyoungki Park* and John W. Wilkins \\ Department of Physics, The Ohio State University, Columbus, Ohio 43210, USA
}

(Received 1 June 2009; published 17 June 2009)

\begin{abstract}
Interstitials stored in $\{311\}$ or $\{111\}$ habit planes form rows of interstitial chains elongated in $\langle 011\rangle$ direction. Exploiting the large aspect ratio to treat chains as infinite, first-principles calculations of large computation supercells reveal a unique formation energy trend for each defect, which is closely correlated with its distinct shape. The most energetically favorable structure changes from $\{311\}$ rodlike defects to Frank loops as the number of interstitials in the defect increases. These results are consistent with transmission electron microscopy studies.
\end{abstract}

DOI: 10.1103/PhysRevB.79.241203

PACS number(s): 61.72.Nn, 61.72.Bb, 61.72.Cc, 71.15.Nc

\section{INTRODUCTION}

From the early 1970s, silicon technology knew that annealing after ion implantation produced extended $\mathrm{Si}$ interstitial defects. Transmission electron microscopy (TEM) reveals three distinct, stable extended interstitial defects whose nucleation, evolution, and stability still challenge us.

Figure 1(a) shows TEM images of rodlike defects ${ }^{1}$ with $\{311\}$ habit planes; also $\{111\}$ rodlike defects exist. Figure 1(b) shows Frank loops ${ }^{2}$-finite faulted $\{111\}$ planes of interstitials. All have large aspect ratios from a few for Frank loops to hundreds for rodlike defects. The rodlike defects can grow up to a micrometer long, but they are typically 10-15 $\mathrm{nm}$ wide. For all, the defected atomic structures can be characterized by the arrangement of interstitial chains and the elongation direction of the defects in Fig. 1 is parallel to the chains running in $\langle 011\rangle$. Figure 2 displays the configurations of the interstitial chains for each defect, where interstitial chains run in/out of paper.

This Rapid Communication presents extensive densityfunctional theory (DFT) calculations for the three defect structures in 3000-plus atom supercells. Using such large supercells ensures the correct stability ordering among defects and greatly extends the range of results for rodlike defects. Moreover, we report DFT results for Frank loops, which are essential to establish the relative stability.

We treat the interstitial chains as infinitely long. The twoatom chain element is repeated by the periodic boundary condition. Relaxation occurs for the remaining two dimensions in Fig. 2. The core structure of the resulting bilayer has a finite number of interstitial chains $I$ uniquely arranged on the habit planes for each defect. Note that structures in Fig. 2 avoid dangling bonds through the formation of five-, seven-, and eight-member rings besides regular six-member rings. The row of interstitial chains terminates with end units $E$, and we measure the width of the defect across two end units in the bilayer.

\section{SUMMARY OF RESULTS}

Figure 3 summarizes results of the extensive DFT calculations within the local-density approximation (LDA). 29 core structures are relaxed for the formation-energy-perinterstitial $E_{\text {int }}^{f}$ trends for three extended defects with increas- ing number of interstitial chains $N(I)$. For up to a dozen interstitial chains, the order of stability, from the most stable, is $\{311\},\{111\}$, and the Frank loop. The $\{311\}$ defect lies more than $0.2 \mathrm{eV} /$ interstitial below either. In contrast to $\{311\}$ and $\{111\}$ defects, the formation energy of the Frank loop decreases continuously with increasing width. This indicates that, eventually, Frank loops with a large number of interstitials become most stable, and the planar structures with comparable dimensions in length and width are energetically favorable. The infinite-planar Frank loop has the energy of $0.03 \mathrm{eV} /$ interstitial that is consistent with the elastic limit for the $\{111\}$ stacking fault. ${ }^{3}$ The infinity limit is calculated with the supercell in which a unit of interstitial chains is repeated by the periodic boundary condition in the direction of width, i.e., no termination with end units.

In Fig. 3, the formation energy trends of $\{311\}$ and $\{111\}$ defects initially drop with the increasing number of interstitial chains and reach minima. The defect with $N(I)=10$ is most stable for the $\{311\}$. The corresponding optimum width is $\sim 10 \mathrm{~nm}$, which agrees with the typical width of rodlike $\{311\}$ defects appearing in TEM images., ${ }^{1,4}$ Table I shows that the most stable rodlike $\{311\}$ defect has the formation energy per interstitial $E_{\min }^{f}=0.12 \mathrm{eV}$, which is substantially lower, by about $0.2 \mathrm{eV}$, than the energy of the infinitely long and wide planar defect $E_{\infty}^{f}$. Furthermore, Table I also shows that the lowest formation energy per interstitial value of
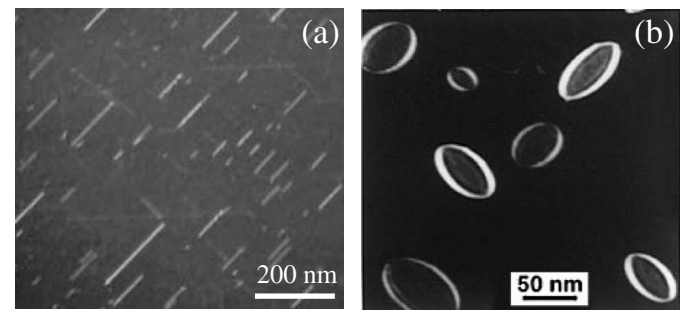

FIG. 1. Plan view, weak-beam, dark-field TEM images of different extrinsic extended defects in silicon: (a) $\{311\}$ rodlike defects (Ref. 1) and (b) Frank loops (Ref. 2). $\{311\}$ are habit planes for interstitials in most rodlike defects, but rodlike defects with $\{111\}$ habit planes have also been observed. Rodlike defects extend in $\langle 011\rangle$. The Frank loop has a piece of faulted interstitial plane in $\{111\}$ habit plane. The extra plane is bounded by a dislocation line, which appears as a bright elliptical line with the large axis parallel to $\langle 011\rangle$ in (b). 


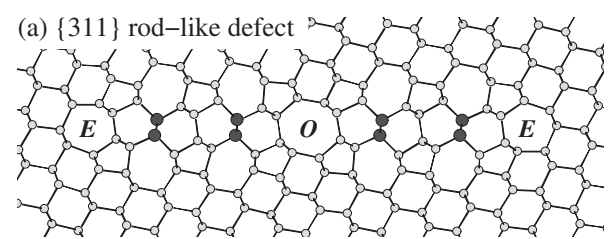

(b) $\{111\}$ rod-like defect
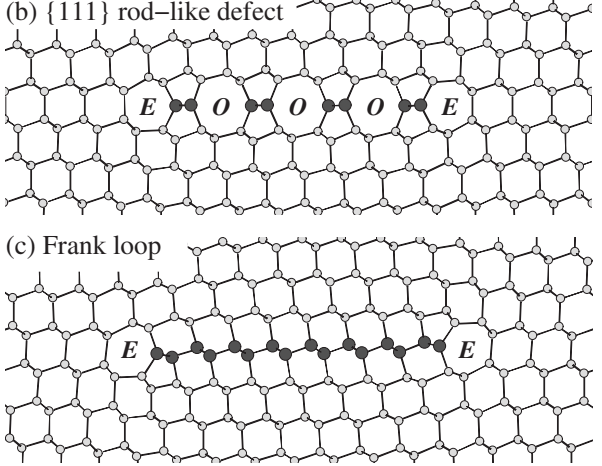

FIG. 2. The core atomic configurations of extended interstitial defects in the $(01 \overline{1})$ bilayer plane-cross-sectional views of defects. The in/out of paper is parallel to the elongation direction for both rodlike defects and Frank loops in Fig. 1. The dark atoms represent interstitial chains running in/out of paper: four interstitial chains are depicted in (a) and (b), eight chains in (c). End units $E$ form boundaries between defects and bulk $\mathrm{Si}$; the separation between $E$ 's determines the width of the defect. In each figure, structures are oriented so that the habit plane horizontally closes two end units and interstitial chains, and extends in [01̄] direction, in/out of paper. (a) The $\{311\}$ rodlike defect is a combination of interstitial chains and eight-member rings $O$. (b) The $\{111\}$ rodlike defect is a regular sequence of double five- and single eight-member rings. (c) The Frank loop connects interstitial chains to form a stacking fault bounded by a dislocation line, i.e., end units $E$ 's.

$\{111\}$ defects is $0.33 \mathrm{eV}$, which is about $0.2 \mathrm{eV}$ higher than the $\{311\}$ value. The probability for an interstitial to be in $\{111\}$ instead of $\{311\}$ defects is about $\exp \left(-0.2 / k_{B} T\right) \approx 0.10-0.15$ for the annealing temperature range of $1000-1200 \mathrm{~K}$. This is consistent with the $10 \%-15 \%$ observed population of $\{111\}$ defects among all rodlike defects in that temperature range. ${ }^{6-8}$

The LDA exchange-correlation energy is chosen to allow comparison with previous works. But tests with the generalized gradient approximation of Perdew and Wang $\left(\right.$ GGA-PW91) ${ }^{9}$ show that the formation energies per interstitial are shifted upward by about a third of eV, but the general trends persist. For four interstitial chains, $N(I)=4$, GGA (LDA) formation energies of the $\{311\}$, the $\{111\}$, and the Frank defects are $0.58(0.25), 0.81(0.47)$, and $1.20(0.87) \mathrm{eV}$, respectively. For infinite-planar structures, $E_{\infty}^{f}$ for the $\{311\}$ and the $\{111\}$ defects are $0.61(0.30)$ and $0.64(0.34) \mathrm{eV}$.

\section{IMPLICATIONS FOR GROWTH}

In an irradiated sample, interstitials aggregate to form small compact clusters first and during subsequent annealing they can grow into larger extended defects. ${ }^{10-14}$ The calcu-

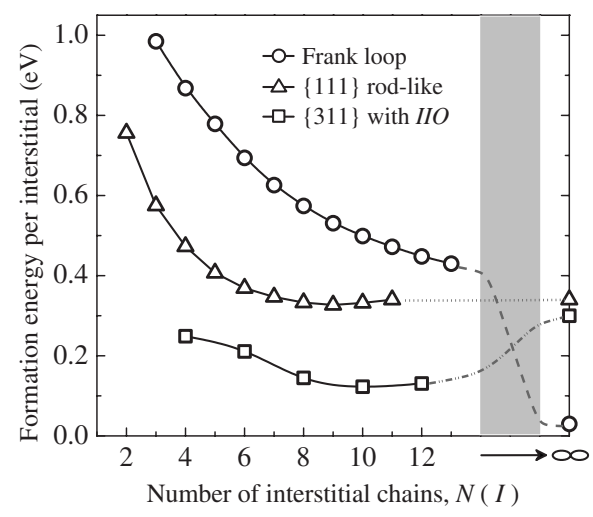

FIG. 3. Relative stability of extended defects with respect to the number of infinitely long interstitial chains enclosed. $\{311\}$ rodlike defects are the most stable structures for a small number of interstitial clusters. As the number of interstitial increases the order of stability changes. The ever decreasing trend eventually makes Frank loops become the most stable ones, with the asymptotic value 0.03 $\mathrm{eV}$. The formation energies with an infinite number of interstitial chains are from the calculations with infinite-planar models, i.e., periodically repeating structures in both width and length directions.

lated energy hierarchy and the interstitial densities in Table I imply that for extended defects, rodlike $\{311\}$ defects are initially favored. If the reaction barrier is not too high and the temperature is high enough to thermally activate the transformation, rodlike defects can evolve into Frank loops that are more stable and compact for a large number of interstitials. This evolution model is consistent with the observed thermal evolution of varying defect structure with the temperature and the duration of annealing. ${ }^{2,15-19}$

\section{COMPUTATIONAL DETAILS}

Defect calculations must use large supercells, so the images generated by periodic boundary conditions do not invalidate results for real defects. Our largest calculations have about 3000 atoms in the supercell. All defect structures are modeled to periodically repeat in $[01 \overline{1}]$ direction, that is, the interstitial chains are infinitely long and the periodicity is

TABLE I. Summary of the minimum formation energy per interstitial $E_{\min }^{f}$ and the energy with an infinite number of chains $E_{\infty}^{f}$ for each defect. $E / I / E$ and $E / I I O / E$ are $\{311\}$ rodlike defect structures with varying number of interstitial chains $I$ and $I I O$ units, respectively. Also listed is the average density of interstitials on the habit planes, $\sigma$, showing that the most densely packed cluster is Frank loop.

\begin{tabular}{lcccc}
\hline \hline Defect structure & $\begin{array}{c}E_{\min }^{f} \\
(\mathrm{eV})\end{array}$ & $\begin{array}{c}E_{\infty}^{f} \\
(\mathrm{eV})\end{array}$ & $\begin{array}{c}\sigma \\
\left(\mathrm{nm}^{-2}\right)\end{array}$ \\
\hline$\{311\}$ defect & $E / I / E$ & 0.33 & 0.51 & 8.1 \\
& $E / I I O / E$ & 0.12 & 0.30 & 5.5 \\
$\{111\}$ defect & 0.33 & 0.34 & 7.9 \\
Frank loop & 0.03 & 0.03 & 16.1 \\
\hline \hline
\end{tabular}


two atom-layers thick, $1 / \sqrt{2}$ in units of the lattice constant $\left(a_{0}=5.43 \AA\right)$. The resulting bilayer ${ }^{20}$ for our largest supercell is about $23 a_{0}$ on a side $\left(33 \times a_{0} / \sqrt{2}\right.$ and $23 \times a_{0}$ along [011] and [100], respectively), about 2.4 times larger than the horizontal dimension of Fig. 2 panels. In the bilayer with the chosen orthogonal supercell directions, [011] and [100], the habit planes are slanted toward the diagonal. This increases the image separation even more in the direction perpendicular to the habit planes, which is crucial for calculations with planar defects. The plane-to-plane separation is about $33 a_{0}$ and the tail-to-tail separation between adjacent images in [011] or [100] directions is always kept greater than $10 a_{0}$. In most cases, we report converged results to $0.01 \mathrm{eV}$ with respect to the supercell size. Even for the largest defect structures, the cell-size errors are within $0.02 \mathrm{eV}$.

For a given number of interstitial chains, the core structures of the $\{111\}$ and the Frank loop are unique, whereas different arrangements of interstitial chains and interrupting eight-member rings make energetically different $\{311\}$ structures. This has been observed ${ }^{21,22}$ and examined ${ }^{23-25}$ with both DFT and tight-binding with a common conclusion: the structure with repeating IIO units is the most stable $\{311\}$ defect, where $I$ represents an interstitial chain and $O$ is an eight-member ring (see Fig. 2). Our results are consistent with this. ${ }^{20}$ Hence, for $\{311\}$ defects, we study the formation energy trend with varying number of $I I O$ units, but also record results of the structure with only $I s$, i.e., no $O$ s, for the sake of comparison in Table I.

The starting structures are obtained by extensive classical molecular-dynamics simulations ${ }^{26}$ of tens-of-thousand-atom supercells. The extracted 3000-atom structures are further relaxed by the conjugate gradient minimization within LDA with the plane-wave cutoff of $260 \mathrm{eV}$ and $1 \times 1 \times 3 k$-point mesh using Vienna $a b$ initio simulation package (VASP). ${ }^{27,28}$ Even with currently available computing resources such as access to powerful supercomputers and codes with greatly improved parallel performance, one DFT relaxation of a 3000-atom supercell still takes a few hundred thousand wall clock hours.

All comparisons of stability are based both on the formation energy per interstitial atom with increasing number of interstitial chains on the habit plane. The formation energy for $N_{\text {int }}$ interstitials and $N_{\text {bulk }}$ bulk atoms is defined by

$$
E^{f}=E_{\text {tot }}\left[N_{\text {int }}+N_{\text {bulk }}\right]-\frac{N_{\text {int }}+N_{\text {bulk }}}{N_{\text {bulk }}} E_{\text {tot }}\left[N_{\text {bulk }}\right],
$$

and the formation energy per interstitial $E_{\text {int }}^{f}=E^{f} / N_{\text {int }}$.

\section{COMPARISON WITH PREVIOUS RESULTS}

This Rapid Communication reports energetics of three distinct extended defects all studied at the same firstprinciples accuracy level. The atomic configurations extracted from MD simulations enable our study of energetics of Frank loops at DFT level. No first-principles studies have been previously done for Frank loops, only elasticity estimations.

The unprecedented large supercells used in our calculations allow defect models with finite width. Previous DFT

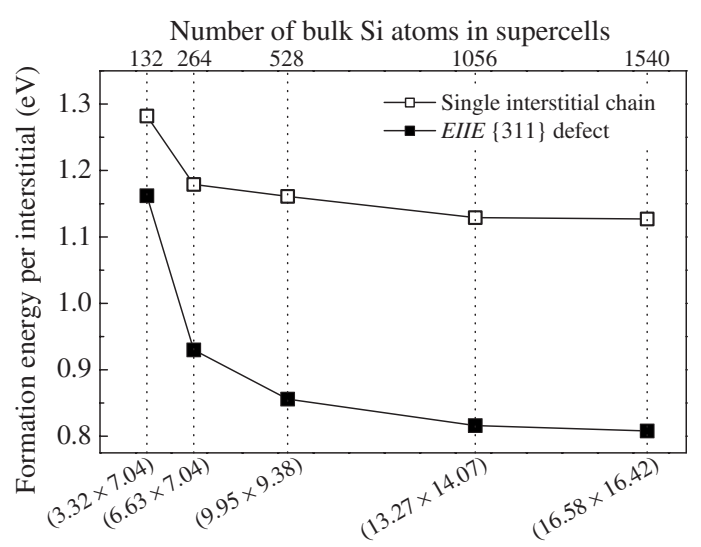

Dimensions of supercells along [311] and [2 $\overline{33}$ ] in unit of $a_{0}$

FIG. 4. Formation energy per interstitial (GGA-PW91) convergence with respect to the supercell size for infinitely long defects with a single interstitial chain and EIIE $\{311\}$. Even for defects with a small number of chains, the converged $E_{\text {int }}^{f}$ to $0.01 \mathrm{eV}$ can only be obtained when supercells with more than 1000 atoms are used due to the long-range strain field.

calculations $^{10,25}$ on $\{311\}$ or $\{111\}$ defects were limited to the infinite-planar defects, ${ }^{10,25}$ or defects with only one or two interstitial chains. ${ }^{13}$ Moreover, the large supercells reduce the finite-size supercell error due to the long-range strain field. Consequently, our calculations show formation-energy values that are always lower than previously calculated ones, ${ }^{10,13,25}$ and establish the correct stability ordering among the three defects. Figure 4 demonstrates the effect of the strain field on the convergence of the formation energy. The distortion from five-, seven-, eight-member rings, and layers of interstitials in defects creates a strain field. Even defects with a small number of interstitial chains produce quite a long-range field. This not only affects the convergence of calculated energies but also energy differences between different defects.

Among three extended defects, $\{311\}$ defects are the most studied in relation to the transient enhanced diffusion phenomenon in ion-implanted samples. Previous LDA calculations $^{10,25}$ of the infinite-planar $\{311\}$ defect reported $0.4-0.7 \mathrm{eV}$. In addition to $\{311\}$ defects, careful TEM analyses uncovered the existence of rodlike defects with $\{111\}$ interstitial habit planes. ${ }^{6,7,15}$ They form less frequently, but the only existing previous LDA calculation ${ }^{25}$ of the infiniteplanar $\{111\}$ defect reported that its formation energy is lower, $0.36 \mathrm{eV}$, than the infinite-planar $\{311\}$ defect. Our calculation shows $0.30 \mathrm{eV}$ for the infinite-planar $\{311\}$ defect. More importantly, we prove that the $\{311\}$ defect energetically favors the rodlike morphology and has the energy 0.12 $\mathrm{eV}$ with the optimum width of $\sim 10 \mathrm{~nm}$. Furthermore, we report that the $\{111\}$ defect structure is always less stable than the $\{311\}$, by ranging from 0.1 to $0.04 \mathrm{eV}$ depending on the number of interstitial chains in defects. Recently, Lee and Hwang $^{13}$ calculated formation energies of defects with a small number of interstitial chains within GGA. Their 1.11 and $1.28 \mathrm{eV}$ for the infinitely long EIIE $\{311\}$ defect and the single interstitial chain are also considerably higher than our GGA values in Fig. 4, 0.81 and $1.12 \mathrm{eV}$.

In conclusion, massively parallelized density-functional relaxations uncover the detailed energetics of three extended 
interstitial defects commonly observed during annealing processes after ion-implantation or high-energy-electron irradiation. The calculated formation energies with varying cluster size reveal a unique trend for each defect, which is closely correlated with their distinct shapes. The $\{311\}$ defect with a narrow rodlike structure is the most stable for a small number of interstitials. However, the most densely packed defect Frank loop is the structure with the lowest formation energy for clusters with a large number of interstitials.

\section{ACKNOWLEDGMENTS}

This work was supported by DOE-Basic Energy Science. Computational resources were provided by NERSC and OSC. We especially thank NERSC for the significant portion of the beta testing time of the Cray XT4 and a large amount of allocation of computing time for this project. We thank Jeongnim Kim and David Richie for their useful discussions. We also greatly appreciate Paul Kent at ORNL for optimizing parallel performance of VASP at NERSC. *hkpark@mps.ohio-state.edu

${ }^{1}$ F. Cristiano, N. Cherkashin, X. Hebras, P. Calvo, Y. Lamrani, E. Scheid, B. de Mauduit, B. Colombeau, W. Lerch, S. Paul, and A. Claverie, Nucl. Instrum. Methods Phys. Res. B 216, 46 (2004).

${ }^{2}$ A. Claverie, B. Colombeau, G. B. Assayag, C. Bonafos, F. Cristiano, M. Omri, and B. Mauduit, Mater. Sci. Semicond. Process. 3, 269 (2000).

${ }^{3}$ F. Cristiano, J. Grisolia, B. Colombeau, M. Omri, B. de Mauduit, A. Claverie, L. F. Giles, and N. E. B. Cowern, J. Appl. Phys. 87, 8420 (2000).

${ }^{4}$ D. J. Eaglesham, P. A. Stolk, H. J. Gossmann, and J. M. Poate, Appl. Phys. Lett. 65, 2305 (1994).

${ }^{5}$ G. Z. Pan, R. P. Ostroumov, L. P. Ren, Y. G. Lian, and K. L. Wang, J. Non-Cryst. Solids 352, 2506 (2006).

${ }^{6}$ C. T. Chou, D. J. H. Cockayne, J. Zou, P. Kringhøj, and C. Jagadish, Phys. Rev. B 52, 17223 (1995).

${ }^{7}$ L. Fedina, A. Gutakovskii, A. Aseev, J. Van Landuyt, and J. Vanhellemont, Philos. Mag. A 77, 423 (1998).

${ }^{8}$ L. Fedina, A. Gutakovskii, A. Aseev, J. Van Landuyt, and J. Vanhellemont, Phys. Status Solidi A 171, 147 (1999).

${ }^{9}$ J. P. Perdew and Y. Wang, Phys. Rev. B 45, 13244 (1992).

${ }^{10}$ J. Kim, F. Kirchhoff, J. W. Wilkins, and F. S. Khan, Phys. Rev. Lett. 84, 503 (2000).

${ }^{11}$ D. A. Richie, J. Kim, S. A. Barr, K. R. A. Hazzard, R. Hennig, and J. W. Wilkins, Phys. Rev. Lett. 92, 045501 (2004).

${ }^{12}$ Y. A. Du, R. G. Hennig, T. J. Lenosky, and J. W. Wilkins, Eur. Phys. J. B 57, 229 (2007).

${ }^{13}$ S. Lee and G. S. Hwang, Phys. Rev. B 78, 045204 (2008).

${ }^{14}$ S. Lee and G. S. Hwang, Phys. Rev. B 77, 085210 (2008).

${ }^{15}$ S. Boninelli, N. Cherkashin, A. Claverie, and F. Cristiano, Appl.
Phys. Lett. 89, 161904 (2006)

${ }^{16}$ L. S. Robertson, K. S. Jones, L. M. Rubin, and J. Jackson, J. Appl. Phys. 87, 2910 (2000).

${ }^{17}$ G. Z. Pan and K. N. Tu, J. Appl. Phys. 82, 601 (1997).

${ }^{18}$ G. Z. Pan, K. N. Tu, and A. Prussin, J. Appl. Phys. 81, 78 (1997).

${ }^{19}$ A. Claverie, B. Colombeau, B. De Mauduit, C. Bonafos, X. Hebras, G. Ben Assayag, and F. Cristiano, Appl. Phys. A: Mater. Sci. Process. 76, 1025 (2003).

${ }^{20}$ Supplementary information is available at http://www.aip.org/ pubservs/epaps.html

${ }^{21}$ S. Takeda, M. Kohyama, and K. Ibe, Philos. Mag. A 70, 287 (1994).

${ }^{22}$ S. Takeda, Jpn. J. Appl. Phys. 30, L639 (1991).

${ }^{23}$ J. Kim, J. W. Wilkins, F. S. Khan, and A. Canning, Phys. Rev. B 55, 16186 (1997).

${ }^{24}$ M. Kohyama and S. Takeda, Phys. Rev. B 51, 13111 (1995).

${ }^{25}$ J. P. Goss, T. A. G. Eberlein, R. Jones, N. Pinho, A. T. Blumenau, T. Frauenheim, P. R. Briddon, and S. Öberg, J. Phys.: Condens. Matter 14, 12843 (2002); There is an inconsistency between results in the table and the conclusion in the text of this reference regarding the lowest-energy $\{311\}$ structure. The formation energy per interstitial of $/ I I O /$ is lower than $/ I I I O /$ by $0.02 \mathrm{eV}$ in the table, which is in agreement with our results (see supplement).

${ }^{26}$ A highly optimized modified embedded atom method potential is used. Results of MD simulations will be discussed in a separate publication.

${ }^{27}$ G. Kresse and J. Hafner, Phys. Rev. B 47, 558(R) (1993).

${ }^{28}$ G. Kresse and J. Furthmüller, Phys. Rev. B 54, 11169 (1996). 